\title{
Extension of Pirogov-Sinai Theory of Phase Transitions to Infinite Range Interactions. II. Phase Diagram
}

\author{
Yong Moon Park \\ Department of Mathematics, Yonsei University, Seoul 120, Korea
}

\begin{abstract}
This paper is the second part of our attempt of an extension of the Pirogov-Sinai theory of phase transitions at low temperatures, applicable to the lattice spin systems with finite range interactions, to the systems with infinite range interactions. Employing the cluster expansion method developed in Part I and modifying the notion of the truncated contour model introduced by Zahradnik, we extend the Pirogov-Sinai result on the structure of phase diagrams to our situations. As an application, we apply our result to Potts models with infinite range interactions.
\end{abstract}

\section{Introduction: Main Result}

We continue our attempt to extend the Pirogov-Sinai (PS) theory of phase transitions to classical (discrete and bounded) lattice spin system with infinite range interactions. In Part I [3], we have developed a cluster expansion method, and shown that under appropriate assumptions on the interactions the cluster expansion converges for each stable ground state. Thus for each stable ground state we can construct via the cluster expansion method an infinite volume limit pure Gibbs state [3]. In this paper we use the cluster expansion method and some modifications of Zahradnik's version of the PS theory [6] to investigate the structure of the phase diagram for a given system. In order to show how our result can be applied, we study the phase diagrams of Potts models with infinite range interactions.

The PS theory applies to general bounded and discrete lattice spin systems of the following types: The particles (spins) interact with arbitrary finite range periodic potentials. The Hamiltonian of the system, $H_{0}$, has $n$ ground states which satisfy the Peierls condition $[4,5]$. Consider the structure of the phase diagram of the Hamiltonian

$$
H_{\lambda}=H_{0}+\sum_{i=1}^{n-1} \lambda_{i} H_{i}
$$

in the $n-1$ dimensional parameter space. The $H_{i}$ are perturbations which lift the

\footnotetext{
* Work supported in part by the Basic Science Research Program, Ministry of Education, 1987
} 
degeneracy of $H_{0}$ and produce, in $\lambda$-space, the following topological structure of the ground states of $H_{\lambda}$ : There are $n$ lines starting from the origin on which $H_{\lambda}$ has $n-1$ periodic ground states, two dimensional surfaces bounded by these lines on which there are $n-2$ ground states, etc. The main result of the PS theory is that for sufficiently low temperatures the phase diagram perfectly mimics the above structure $[4,5]$.

We wish to extend the PS result to the systems with infinite range interactions. The PS theory is based on a contour model and a contour model with parameters. For infinite range interactions, we are unable to relate the models to any type of interacting contour models with parameters. On the other hand, the notion of the truncated contour model introduced by Zahradnik [6] can be extended to the systems, and the cluster expansion method for the truncated model can be developed as in Part I. Thus we combine the cluster expansion method and suitable modifications of Zahradnik's approach of the PS theory [6] to extend the PS result to our case. Following the main idea of Zahradnik [6] together with the method used in this paper, it can be possible to extend the result on the completeness of the phase diagram to our situation, but we do not check this. As we said in Part I, we believe that our result can also be extended to continuous lattice spin systems [2] and to Widom-Rowlinson models [1].

Let us briefly describe our result. Throughout this paper we will adapt the notations and the definitions used in Part I [3]. Let $\mathbb{Z}^{v}, v \geqq 2$, be the $v$-dimensional lattice space and let $\Omega$ be the finite set of spin values. For any $\Lambda \subset \mathbb{Z}^{v}$, denote by $\Omega^{\Lambda}$ the set of all configurations on $\Lambda$. For any $\omega \in \Omega^{\mathbb{Z}^{v}}$, denote by $\omega_{\Lambda}$ the restriction of $\omega$ on $\Lambda$. We write the constant configuration $\omega(x)=q, x \in \mathbb{Z}^{v}$, as $\omega_{q}$, and the constant configuration on $\Lambda$ as $\omega_{\Lambda, q}$. As in $I$, we use the norm $\|x\|=\max _{1 \leqq l \leqq v}\left|x_{i}\right|, x \in \mathbb{Z}^{v}$. For a fixed real number $s \geqq 1$, let some family $\left\{\Phi_{A}\right\}$ of finite range interactions be given, invariant with respect to shifts in $\mathbb{Z}^{v}$ and with the finite interaction radius $s\left(\Phi_{A}=0\right.$ if $\operatorname{diam}(A)>s)$. For any $x, y \in \mathbb{Z}^{v}$, let $J_{x-y}: \Omega \times \Omega \rightarrow \mathbb{R}$ be a symmetric function. For a finite $\Lambda \subset \mathbb{Z}^{v}$ and a configuration $\omega \in \Omega^{\mathbb{Z}^{v}}$, the Hamiltonian is given by

$$
H\left(\omega_{\Lambda} \mid \omega_{\Lambda^{c}}\right)=\sum_{\substack{A \cap \Lambda \neq \phi \\ \operatorname{diam}(A) \leqq s}} \Phi_{A}\left(\omega_{A}\right)+\sum_{\{x, y\} \cap \Lambda \neq \phi} J_{x-y}(\omega(x), \omega(y)) .
$$

A decay property on the two-body potential $J_{x-y}$ has been imposed in Assumption 2.3.2 of Part I.

For a given (fixed) subset $Q \subset \Omega$ and $s \geqq 1$, the $q$-contours $\Gamma^{q}, q \in Q$, and the external $q$-contour systems $\partial=\left\{\Gamma_{1}^{q}, \ldots, \Gamma_{n}^{q}\right\}$ have been defined in Sect. 2.1 of the Part I. For any $q$-contour $\Gamma=\left(M, \omega_{M}\right)$, let

$$
\begin{aligned}
\Phi(\Gamma)= & \sum_{\substack{A \cap M \neq \phi \\
\operatorname{diam}(A) \leqq s}} \frac{|A \cap M|}{|A|}\left\{\Phi_{A}\left(\omega_{A}\right)-\Phi_{A}\left(\omega_{A, q}\right)\right\} \\
& +\sum_{\substack{\{x, y\} \cap M \neq \phi \\
\|x-y\| \leqq s}} \frac{|\{x, y\} \cap M|}{2}\left\{J_{x-y}(\omega(x), \omega(y))-J_{x-y}(q, q)\right\} .
\end{aligned}
$$

We now restate the basic assumptions in Assumption 2.3.1 and Assumption 2.3.2 in Part I: 
Assumption 1.1. [Peierls condition] For any $q$-contour $\Gamma=\left(M, \omega_{M}\right), q \in Q$, there exists a sufficiently large $\tau>0$ such that $\Phi(\Gamma) \geqq \tau|\Gamma|$ holds.

Assumption 1.2. [Regularity condition] The two-body potential satisfies

$$
\sup _{\omega \in \Omega}\left|J_{x-y}(\omega(x), \omega(y))\right| \leqq e^{-\delta(x, y)} J(\|x-y\|)
$$

where $\delta$ is a metric on $\mathbb{Z}^{v}$ such that

$$
\sup _{x} \sum_{y \in \mathbb{Z}^{v}} e^{-\delta(x, y)} \leqq M<\infty
$$

for some constant $M>0$, and $J: \mathbb{R} \rightarrow \mathbb{R}_{+}$is a non-negative function satisfying

$$
2 \sum_{r=0} \sum_{y \in \mathbb{Z}^{v}} J(2 s+r+\|y\|) \leqq \tau / 48
$$

for the positive real number $s$ introduced in the definition of contours.

Remarks. (a) Since we have absorbed $\beta=1 / T$ into the Hamiltonian, the parameter $\tau$ is proportional to $\beta$.

(b) If we are only interested in the maximum number of pure states, a weaker condition $(\delta(x, y) \rightarrow \infty$ as $\|x-y\| \rightarrow \infty)$ than condition (1.4) on the metric $\delta$ will be sufficient. See the remark below Assumption 2.3.2 of Part I.

(c) In applications, one may first choose the parameter $s(\geqq 1)$ such that (1.5) and the Peierls condition are satisfied, and then choose $\beta$ sufficiently large so that $\tau$ becomes large (See Sect. 5).

We now state the main theorem in this paper. For a given Hamiltonian $H$ of the form (1.1) and for any $q \in Q$, we write the specific energy by

$$
e_{q}(H)=\sum_{0 \in A} \frac{1}{|A|} \Phi_{A}\left(\omega_{A, q}\right)+\frac{1}{2} \sum_{x \in \mathbb{Z}^{\nu}} J_{x}(q, q) .
$$

Then we have the following result:

Theorem 1.3. Let $H_{0}$ be some translational invariant Hamiltonian of the form (1.1) and let $H_{1}, \ldots, H_{n-1}$ be another Hamiltonian of the same type. Consider the family

$$
\left\{H_{\lambda}=H_{0}+\sum_{i=1}^{n-1} \lambda_{i} H_{i}: \lambda=\left(\lambda_{1}, \ldots, \lambda_{n-1}\right) \in \mathscr{U}\right\},
$$

where $\mathscr{U}$ is some neighborhood of zero in $\mathbb{R}^{n-1}$. Suppose that there is a fixed family $\left\{\omega_{q}: q \in Q\right\}, Q \subset \Omega,|Q|=n$, of constant configurations such that the following conditions are satisfied.

(i) All $e_{q}\left(H_{0}\right), q \in Q$, are the same.

(ii) Let $q_{1}, \ldots, q_{n}$ be some ordering of $Q$. Then the vectors $e_{i}=\left(e_{q_{1}}\left(H_{i}\right), \ldots, e_{q_{n}}\left(H_{i}\right)\right)$ are independent and their linear span $\mathbf{L}$ does not contain the vector $(1,1, \ldots, 1)$ (degeneracy removing assumption).

(iii) The Peierls condition in Assumption 1.1 is satisfied for all $q \in Q$ and all $H_{\lambda}$, $\lambda \in \mathscr{U}$.

Furthermore assume that the regularity condition in Assumption 1.2 is satisfied for all $H_{\lambda}, \lambda \in \mathscr{U}$. 
Then there is a neighborhood of zero $\mathscr{V} \subset\left(\mathbb{R}^{n}\right)^{0} \equiv\left\{h^{0}: h^{0}=\left(h_{q_{1}}^{0}, \ldots, h_{q_{n}}^{0}\right)\right.$, min $\left.h_{q_{i}}^{0}=0\right\}$ and a homeomorphism $\lambda \in \mathscr{U} \rightarrow h^{0}(\lambda) \in \mathscr{V}$ with the following property: Every $q_{i} \in Q$ such that $h^{0}(\lambda)_{q_{1}}=0$ is stable. Thus for every $\lambda \in \mathscr{U}$ and $q \in Q(\lambda)=$ $\left\{q \in Q(\lambda)=\left\{q \in Q: h^{0}(\lambda)_{q}=0\right\}\right.$, one can construct a pure Gibbs state $\rho_{\lambda}^{q}$ on $\Omega^{\mathbb{Z}^{v}}$ by the cluster expansion method.

The above is the extension of the PS theory to infinite range interactions. This paper is devoted to the proof of the above theorem.

Let us describe briefly the general strategy of the proof and also outline the contents of the rest of this paper. Recall from Part I that an external $q$-contour $\Gamma$ is stable if

$$
e^{-\Psi(\Gamma)} \equiv\left\{Z_{q}(\Gamma) / Z_{q}(\operatorname{Int} \Gamma)\right\} \leqq e^{--\tau|\Gamma| / 3}
$$

A given ground state $q \in Q$ is said to be stable if every $q$-contour is stable (Definition 2.3.3 of I). We have shown for every stable $q \in Q$ that the infinite volume limit contour correlation functions exist and satisfy the cluster property (Theorem 2.3.4 of Part I). Using the result together with a simple argument one may construct a pure Gibbs state on $\Omega^{\mathbb{Z}^{v}}$ for every stable $q \in Q$. Thus the problem reduces to a study of the structure of stable ground states for $\lambda \in \mathscr{U}$. Using the Peierls condition we will relate the stability condition (1.7) to the bounds on $Z_{q}(B) / Z_{q^{\prime}}(B), B$ $\subset \mathbb{Z}^{v}, q, q^{\prime} \in Q$ (Proposition 4.2). In Sect. 2, we introduce truncated partition functions and truncated contour correlation functions which are modifications of Zahradnik's notion [6]. We then use the cluster expansion method developed in Part I to establish a continuity property of free energy of the truncated model (Proposition 3.2). In Sect. 4 we use Zahradnik's method [6] and the cluster expansion method to obtain upper and lower bounds on the partition function $Z_{q}(B), B \subset \mathbb{Z}^{v}$ and $q \in Q$ (Theorem 4.1). Then the proof of Theorem 1.3 will follow from the method used in Zahradnik [6].

In order to give a general idea how our result can be applied to a specific model, we investigate the phase diagram for Potts models with infinite range interactions in Sect. 5. Appendix is devoted to a proof of Proposition 4.2.

\section{Truncated Partition Functions and Truncated Contour Correlation Functions}

In this section we introduce a truncated model which is a modification of Zahradnik's notion [6], and then we establish basic properties of the model. Recall notations and definitions from Part I: For any finite $\Lambda \subset \mathbb{Z}^{v}$ and a fixed $q \in Q$, let $\mathscr{C}_{q}(\Lambda)$ and $\mathscr{E}_{q}(\Lambda)$ be the set of finite $q$-contours and the family of external $q$-contour systems respectively. For a $\Gamma \in \mathscr{C}_{q}(\Lambda)$, denote by $\Omega(\Gamma)$ the set of all configurations $\omega_{V(\Gamma)}$ on $V(\Gamma)=\operatorname{Supp} \Gamma \cup \operatorname{Int} \Gamma$, which satisfying the condition that being extended by $q$ to whole $\mathbb{Z}^{v}$, they have $\Gamma$ as its external $q$-contour. Then the partition function and contour correlation functions have been defined by

and

$$
Z_{q}(\Lambda)=\sum_{\partial \in \mathscr{\sigma}_{q}(\Lambda)} \int_{\partial} d \omega d^{-H(\hat{\partial} ; \omega)},
$$

$$
\rho_{\Lambda, q}(\partial)=\frac{1}{Z_{q}(\Lambda)} \sum_{\hat{\partial}^{\prime} \in \mathscr{E}_{q}(\Lambda \backslash V(\partial))} \int_{\partial \cup \hat{\partial}^{\prime}} d \omega e^{-H(\hat{\partial} ; \omega)}
$$


where for any $\partial=\left\{\Gamma_{1}, \ldots, \Gamma_{n}\right\} \in \mathscr{E}_{q}(\Lambda), \int_{\hat{c}} d \omega=\sum_{\omega \in \Omega\left(\Gamma_{1}\right)} \ldots \sum_{\omega \in \Omega\left(\Gamma_{n}\right)}$, and the contour Hamiltonian $H(\partial ; \omega)$ is of the form:

$$
H(\partial ; \omega)=\sum_{\Gamma \in \partial} \Phi_{1}(\Gamma ; \omega)+\sum_{\Gamma, \Gamma^{\prime} \in \partial} \Phi_{2}(\Gamma ; \omega)
$$

For the details, see Chapter 2 of Part I. For a $\Gamma \in \mathscr{C}_{q}(\Lambda)$, the crystal partition function has been given by

$$
Z_{q}(\Gamma)=\int_{\Gamma} d \omega e^{-\Phi_{1}(\Gamma ; \omega)}
$$

We define the refined contour correlation functions by

$$
\rho_{\Lambda, q}(\partial ; \omega)=\frac{1}{Z_{q}(\Lambda)_{\left.\hat{\sigma}^{\prime} \in \mathscr{E}_{q}(\Lambda \backslash V(\partial))\right)}} \int_{\hat{o}^{\prime}} d \omega^{\prime} e^{-H\left(\partial \cup \hat{o}^{\prime} ; \omega \cup \omega^{\prime}\right)}
$$

for any $\partial \in \mathscr{E}(\Lambda)$ and $\omega \in \Omega(\partial)=\bigcup_{\Gamma \in \partial} \Omega(\Gamma)$. It then follows from (2.2) and (2.5) that

$$
\rho_{\Lambda, q}(\partial)=\int_{\partial} d \omega \rho_{\Lambda, q}(\partial ; \omega)
$$

Let us discuss the refined contour correlation functions in more detail. Let $(\partial ; \omega)$ be given where $\partial \in \mathscr{E}$ and $\omega \in \Omega(\partial)$. Then $(\partial ; \omega)$ corresponds to a (not necessarily external) contour system $\partial^{\prime}=\left\{\Gamma_{1}, \ldots, \Gamma_{n}\right\}$ such that $\partial\left(\subset \partial^{\prime}\right)$ is the set of all external contours in $\partial^{\prime}$. Thus the refined contour correlation functions in this paper correspond to the original contour correlation functions in the PS theory.

From now on we fix a ground state $q \in Q$ and delete $q$ from the notation if there is no confusion involved. For any $\partial, \partial^{\prime} \in \mathscr{E}$ with $\partial \cup \partial^{\prime} \in \mathscr{E}$, let $A\left(\partial^{\prime},(\partial ; \omega)\right)$ be defined as in (3.2.11) of Part I [3]. Since

$$
\rho_{\Lambda}(\partial)=\sum_{\partial^{\prime} \in \mathscr{E}(\Lambda \backslash V(\partial))} \int_{\partial} d \omega A\left(\partial^{\prime},(\partial ; \omega)\right) g_{\Lambda}\left(\partial \cup \partial^{\prime}\right)
$$

by (3.2.14), it is easy to show that for any $\partial \in \mathscr{E}(\Lambda)$ and $\omega \in \Omega(\partial)$,

$$
\rho_{\Lambda}(\partial ; \omega)=\sum_{\partial^{\prime} \in \mathscr{E}(\Lambda \backslash V(\partial))} A\left(\partial^{\prime},(\partial ; \omega)\right) g_{\Lambda}\left(\partial \cup \partial^{\prime}\right) .
$$

We then have the following results:

Proposition 2.1. Let the conditions in Assumption 1.1 and Assumption 1.2 be satisfied and let $q \in Q$ be stable. Then for sufficiently large $\tau$ the infinite volume limit

$$
\rho_{q}(\partial ; \omega)=\lim _{\Lambda \rightarrow \mathbb{Z}^{v}} \rho_{\Lambda, q}(\partial ; \omega)
$$

exists for any $\partial \in \mathscr{E}_{q}$ and $\omega \in \Omega(\partial)$. Furthermore the bound

$$
\rho_{\Lambda}(\partial ; \omega) \leqq e^{\tau|\partial| / 6} K(\partial ; \omega)
$$

holds uniformly in $\Lambda$ for each $\partial \in \mathscr{E}$.

Proof. Employing the method used in the proof of Proposition 4.2.5(b) in Part I, one may show that the bound

$$
\sum_{\hat{o}^{\prime} \in \mathscr{E}(V \backslash V(\hat{o}))}\left|A\left(\partial^{\prime},(\partial ; \omega)\right)\right| e^{\tau\left|\partial \cup \hat{o}^{\prime}\right| / 8} \leqq \text { const. } e^{\tau|\partial| / 8} K(\partial ; \omega)
$$


holds uniformly in $\Lambda$. Thus as in the proof of Theorem 4.1.1(a) of Part I, one can prove that (2.8) is summable absolutely and uniformly in $\Lambda$. The proposition follows as a consequence of (2.8) and Theorem 4.1.4 (and Lemma 4.2.3) of Part I.

Let us give a comment on the construction of pure Gibbs states for stable ground states. As mentioned before, the refined correlation functions $\rho(\partial ; \omega)$ correspond to the contour correlation functions in the PS theory. Thus, the construction of pure states can be done by using Proposition 2.1, Theorem 4.1.1-Theorem 4.1.2 of Part I, and the argument used in [5].

We now introduce a truncated model for infinite range interactions by modifying Zahradnik's notion in [6], and then obtain basic properties of the model. As in [6] we define the level of any contour $\Gamma$ as follows: Define the level of any (not necessary external) contour $\Gamma$ as a maximal number $n$ such that there is some sequence of contours $\Gamma_{1}=\Gamma, \Gamma_{2}, \ldots, \Gamma_{n}$ such that $\operatorname{supp} \Gamma_{i+1} \subset \operatorname{Int}\left(\Gamma_{i}\right)$. Define the level of $\Lambda$ $\subset \mathbb{Z}^{v}$ as a maximal level of any contour $\Gamma$ such that $V(\Gamma) \subset \Lambda$. For given $\Gamma \in \mathscr{C}$ and $\Lambda \subset \mathbb{Z}^{v}$, denote by $\ell(\Gamma)$ and $\ell(\Lambda)$ the levels of $\Gamma$ and $\Lambda$ respectively.

For any external $q$-contour $\Gamma$, let $\Psi(\Gamma)$ be the contour functional defined as in (1.6):

$$
Z_{q}(\Gamma) / Z_{q}(\operatorname{Int} \Gamma)=e^{-\Psi(\Gamma)} .
$$

We define a truncated contour functional $\Psi^{t}(\Gamma)$ as

$$
\Psi^{t}(\Gamma)=\max (\Psi(\Gamma), \tau|\Gamma| / 3) .
$$

Thus an external $q$-contour is stable is $\Psi^{t}(\Gamma)=\Psi(\Gamma)$. For any $\Gamma \in \mathscr{C}, \omega \in \Omega(\Gamma)$ and for any real number $u \geqq 1$, denote

$$
\Phi_{1}^{t}(\Gamma, u ; \omega)=\Phi_{1}(\Gamma ; \omega)+u \Psi^{t}(\Gamma)-\Psi(\Gamma),
$$

and for any $\partial \in \mathscr{C}$ and $\omega \in \Omega(\partial)$, denote

$$
H^{t}(\partial, u ; \omega)=\sum_{\Gamma \in \hat{\partial}} \Phi^{t}(\Gamma, u ; \omega)+\sum_{\Gamma, \Gamma^{\prime} \in \hat{\partial}} \Phi_{2}\left(\Gamma, \Gamma^{\prime} ; \omega\right) .
$$

Notice that from (2.4), (2.9) and (2.11) it follows that

$$
\int_{\Gamma} d \omega e^{-\Phi_{1}^{t}(\Gamma, u ; \omega)} / Z(\operatorname{Int} \Gamma)=e^{-u \Psi^{t}(\Gamma)}
$$

for any $\Gamma \in \mathscr{C}$.

We now define truncated partition functions and truncated contour correlation functions inductively. Let $\Lambda$ have zero level (no contour can be in $\Lambda$ ). In this case the truncated partition function $Z_{q}^{t}(\Lambda, u)$ is defined to be one:

$$
Z_{q}^{t}(\Lambda, u) \equiv Z_{q}(\Lambda)=1 \quad \text { if } \quad \ell(\Lambda)=0 .
$$

Let $\Lambda$ have $n$-level $(\ell(\Lambda)=n)$. Then truncated partition function is defined by

$$
Z_{q}^{t}(\Lambda, u)=\sum_{\partial \in \delta_{q}^{g}(\Lambda) \partial} \int_{\partial} d \omega e^{-H^{t}(\hat{\partial}, u ; \omega)} \prod_{\Gamma \in \partial}\left\{Z_{q}^{t}(\operatorname{Int} \Gamma, u) / Z_{q}(\operatorname{Int} \Gamma)\right\},
$$

where the term corresponding to $\partial=\phi$ equals to 1 . Notice that, if $\ell(\Lambda)=n$, then $\ell(\operatorname{Int} \Gamma) \leqq n-1$ for any $\Gamma \in \mathscr{C}_{q}(\Lambda)$. We define the truncated contour correlation 
functions by

$$
\rho_{\Lambda, q}^{t}(\partial, u)=\frac{1}{Z_{q}^{t}(\Lambda, u)} \sum_{\hat{c}^{\prime} \in \delta_{q}(\Lambda \backslash V(\hat{c}))} \int_{\hat{c} \cup \hat{c}^{\prime}} d \omega e^{-H^{t}\left(\hat{c} \cup \hat{c}^{\prime}, u ; \omega\right)} \prod_{\Gamma \in \hat{c} \cup \hat{c}^{\prime}} \frac{Z_{q}^{t}(\operatorname{Int} \Gamma, u)}{Z_{q}(\operatorname{Int} \Gamma)}
$$

for any $\partial \in \mathscr{E}_{q}(\Lambda)$ and $u \geqq 1$. Since $H(\partial ; \omega) \leqq H^{t}(\partial, u ; \omega)$ for any $u \geqq 1$, it follows from (2.1) and (2.14) that

$$
Z_{q}^{t}(\Lambda, u) \leqq Z_{q}(\Lambda)
$$

For any $\Gamma \in \mathscr{C}_{q}(\Lambda)$ we also define the truncated crystal partition function by

$$
Z_{q}^{t}(\Gamma, u)=\int_{\Gamma} d \omega e^{-\phi_{1}^{t}(\Gamma, u ; \omega)}\left[Z_{q}^{t}(\operatorname{Int} \Gamma, u) / Z_{q}(\operatorname{Int} \Gamma)\right]
$$

For a notational simplification we write

$$
\begin{gathered}
Z_{q}^{t}(\Lambda) \equiv Z_{q}^{t}(\Lambda, u=1), \\
Z_{q}^{t}(\Gamma) \equiv Z_{q}^{t}(\Gamma, u=1), \\
\rho_{\Lambda, q}^{t}(\partial) \equiv \rho_{\Lambda, q}^{t}(\partial, u=1) .
\end{gathered}
$$

It is easy to check that, if there are no infinite range interactions, i.e., $\Phi_{2}\left(\Gamma, \Gamma^{\prime}\right.$; $\omega)=0$, then the above definitions reduce to those in Zahradnik [6].

We next derive a cluster expansion for the truncated model. For $\partial, \partial^{\prime} \in \mathscr{E}_{q}$, let

$$
\begin{aligned}
K_{u}^{t}(\partial ; \omega) & =e^{-H^{t}(\hat{\partial}, u ; \omega)} / \prod_{\Gamma \in \hat{\partial}} Z(\operatorname{Int} \Gamma), \\
K_{u}^{t}\left(\partial, \partial^{\prime} ; \omega\right) & =K_{u}^{t}(\partial ; \omega) \prod_{\Gamma \in \hat{\partial}^{\prime}} g(\partial, \Gamma ; \omega),
\end{aligned}
$$

where $g(\partial, \Gamma ; \omega)$ has been given in (3.1.9) of I. Let $K_{u}^{t}\left(\partial_{1}, \ldots, \partial_{n} ; \omega\right)$ and $K_{u}^{t}\left(\partial_{1}, \ldots, \partial_{n}\right)$ be defined by replacing $K(\partial ; \omega)$ by $K_{u}^{t}(\partial ; \omega)$ (and $\widetilde{K}\left(\partial, \partial^{\prime} ; \omega\right)$ by $\widetilde{K}_{u}^{t}\left(\partial, \partial^{\prime} ; \omega\right)$ ) in the definitions of $K\left(\partial_{1}, \ldots, \partial_{n} ; \omega\right)$ and $K\left(\partial_{1}, \ldots, \partial_{n}\right)$ in (3.2.7) of Part I [3] respectively. Denote also that

$$
g_{\Lambda, u}^{t}(\partial)=\frac{Z^{t}(\Lambda \backslash V(\partial), u) \prod_{\Gamma^{\in} \in \partial} Z^{t}(\operatorname{Int} \Gamma, u)}{Z^{t}(\Lambda, u)} .
$$

Then, using the method employed in Sect. 3.2 and Sect. 3.3 of Part I we obtain a cluster expansion for the truncated model:

$$
\begin{aligned}
\rho_{\Lambda}^{t}(\partial, u) & =K_{u}^{t}(\partial) g_{\Lambda, u}^{t}(\partial)+\sum_{n=1}^{\infty} \sum_{\left\{\partial_{1}, \ldots, \hat{o}_{n}\right\}}^{\prime} K_{u}^{t}\left(\partial, \partial_{1}, \ldots, \partial_{n}\right) g_{\Lambda, u}^{t}\left(\partial \cup\left(\bigcup_{i=1}^{n} \partial_{i}\right)\right), \\
g_{\Lambda, u}^{t} & =\mathbf{1}+\chi_{\Lambda} \mathbf{K}_{u}^{t} \chi_{\Lambda} g_{\Lambda, u}^{t},
\end{aligned}
$$

where the operator $\mathbf{K}_{u}^{t}$ is defined by replacing $K(\partial ; \omega)$ by $K_{u}^{t}(\partial ; \omega)$ (and $\tilde{K}\left(\partial, \partial^{\prime} ; \omega\right)$ by $\left.\tilde{K}_{u}^{t}\left(\partial, \partial^{\prime} ; \omega\right)\right)$ in the definition of $\mathbf{K}$ in Sect. 3.3 of Part $\mathbf{I}$.

The following are the results analogous to those in Theorem 4.1.1Theorem 4.1.4 in Part I, and Proposition 2.2.

Theorem 2.2. Let the conditions in Assumption 1.2 be satisfied. Then for sufficiently large $\tau$ the following results hold for any $q \in Q$ and $u \geqq 1$ : 
(a) The cluster expansion in (2.21) is summable absolutely, uniformly in $\Lambda$. Furthermore the bound

$$
\rho_{\Lambda}^{t}(\partial, u) \leqq e^{-u \tau|\partial| / 6}
$$

holds for any $\partial \in \mathscr{E}_{q}$, uniformly in $\Lambda$.

(b) The infinite volume limit

$$
\rho^{t}(\partial, u)=\lim _{\Lambda \rightarrow \mathbb{Z}^{v}} \rho_{\Lambda}^{t}(\partial, u)
$$

exists for each $\partial \in \mathscr{E}_{q}$. Furthermore the bound

$$
\left|\rho_{\Lambda}^{t}(\partial, u)-\rho^{t}(\partial, u)\right| \leqq \exp \left(-\frac{u \tau}{6}|\partial|-\delta\left(\partial, \Lambda^{c}\right)\right)
$$

holds for any $\partial \in \mathscr{E}_{q}$ and $\Lambda \subset \mathbb{Z}^{v}$.

(c) The cluster property for $\rho^{t}(\partial, u)$ holds.

Theorem 2.3. Under the assumptions as in Theorem 2.3, the following results hold for any $q \in Q$ and $u \geqq 1$ :

(a) The infinite volume limit

$$
g_{u}^{t}(\partial)=\lim _{\Lambda \rightarrow \mathbb{Z}^{v}} g_{\Lambda, u}^{t}(\partial)
$$

exists for each $\partial \in \mathscr{E}_{q}$ and satisfies the integral equation $g_{u}^{t}=\mathbf{1}+\mathbf{K}_{u}^{t} g_{u}^{t}$ on the Banach space $\mathscr{F}_{\xi}, \xi=e^{u \tau / 8}$.

(b) The bound

$$
\left|g_{\Lambda, u}^{t}(\partial)-g_{u}^{t}(\partial)\right| \leqq \exp \left(\frac{u \tau}{8}|\partial|-\delta\left(\partial, \Lambda^{c}\right)\right)
$$

holds for any $\Lambda \subset \mathbb{Z}^{v}$ and $\partial \in \mathscr{E}_{q}$.

Proofs of Theorem 2.2 and Theorem 2.3. Using (2.19), (2.12), the bound (4.2.1) of Part I and (2.13) (in that order) we obtain

$$
\begin{aligned}
K_{u}^{t}(\partial) & \equiv \int_{\hat{c}} d \omega K_{u}^{t}(\partial ; \omega) \leqq\left\{\prod_{\Gamma \in \partial} \int_{\Gamma} d \omega e^{-\Phi_{1}^{t}(\Gamma, u ; \omega)} / Z(\operatorname{Int} \Gamma)\right\} e^{\tau|\partial| / 48} \\
& =\prod_{\Gamma \in \hat{\partial}} \exp \left(-u \Psi^{t}(\Gamma)+\tau|\Gamma| / 48\right) \leqq \exp (-11 u \tau|\partial| / 48)
\end{aligned}
$$

for any $u \geqq 1$, The above is the bound analogous to that in (4.2.13) of Part I. Thus replacing $K(\partial ; \omega)$ in Sect. 4.2-4.4 of Part I by $K_{u}^{t}(\partial ; \omega)$ and using $(2.22)$ in the places where (4.2.13) of I has been used, we prove Theorem 2.2 and Theorem 2.3 by methods the same as those in the proofs of Theorem 4.1.1-Proposition 4.1.4 of Part I. We leave the details to the reader.

\section{Free Energy and Surface Tension for the Truncated Model}

We establish various properties of the free energy (the pressure) and the surface tension for the truncated model such as the existence and continuity property of the free energy. For any finite $\Lambda \subset \mathbb{Z}^{v}$ and $q \in Q$, the finite volume free energy for the 
truncated model is defined by

$$
s_{\Lambda, q}=|\Lambda|^{-1} \log Z_{q}^{t}(\Lambda)
$$

where $Z_{q}^{t}(\Lambda)$ is the truncated partition function $Z_{\Lambda}^{t}(\Lambda, u=1)$. We then have the following result:

Proposition 3.1. Let the regularity condition in Assumption 1.2 be satisfied. For sufficiently large $\tau$ and for each $q \in Q$, the limit

$$
S_{q}=\lim _{\Lambda \rightarrow \mathbb{Z}^{v}} s_{\Lambda, q}
$$

exists in the sense of van-Hove, and $s_{q} \rightarrow 0$ as $\tau \rightarrow \infty$. Furthermore there is a constant $c=c(\tau)$ such that $c \rightarrow 0$ as $\tau \rightarrow \infty$ and for each finite $\Lambda \subset \mathbb{Z}^{v}$ the bound

$$
\left|\log Z_{q}^{t}(\Lambda)-s_{q}\right| \Lambda|| \leqq c(\tau)|\operatorname{bd}(\Lambda)|
$$

holds.

We postpone the proof of the above result to later. We will use the following norm of the Hamiltonians of the type (1.1):

$$
\|H\|=\sum_{\substack{0 \in A \\ \operatorname{diam}(A) \leqq s}} \frac{1}{|A|}\left\|\Phi_{A}\right\|_{\infty}+\sup _{x, \omega} \sum_{y \in \mathbb{Z}^{y}} \frac{1}{2}\left|J_{x-y}(\omega(x), \omega(y))\right| .
$$

We then have the following result:

Proposition 3.2. Let $\left\{H_{\lambda}=H_{0}+\lambda \tilde{H}: \lambda \in \mathscr{U}(0) \subset \mathbb{R},\|\tilde{H}\|=1\right\}$ be a family of Hamiltonians of the type (1.1) satisfying the Peierls condition and the regularity condition. Denote $s_{q}(\lambda)$ the free energy of the truncated model corresponding to the Hamiltonian $H_{\lambda}, u=1$ and $a q \in Q$. Then one-side derivative $(d / d \lambda)( \pm) s_{q}(\lambda)$ exists and satisfies the bound

$$
\left|\frac{d}{d \lambda( \pm)} s_{q}(\lambda)\right| \leqq \varepsilon(\tau)
$$

where $\varepsilon(\tau) \rightarrow 0$ as $\tau \rightarrow \infty$.

In the rest of this section we will prove Proposition 3.1 and Proposition 3.2.

In order to show the above results we need a technical lemma.

Lemma 3.3. Under the assumptions as in Proposition 3.1, the bound

$$
\left|\Psi^{t}(\Gamma)\right|+\left(\left|\frac{d}{d u} Z_{q}^{t}(\operatorname{Int} \Gamma, u)\right| / Z_{q}^{t}(\operatorname{Int} \Gamma, u)\right) \leqq \tau|V(\Gamma)|^{2}
$$

holds for each $q \in Q$ and $\Gamma \in \mathscr{C}_{q}$.

Proof. We will show the bound by using an induction argument on the level of Int $\Gamma$. By the definition of $\Psi^{t}(\Gamma)$ in (2.10) and (2.9) it is easy to show that the bound

$$
\left|\Psi^{t}(\Gamma)\right| \leqq \max \{2\|H\||V(\Gamma)|, \quad(\tau / 3)|V(\Gamma)|\} \leqq c \tau|V(\Gamma)|
$$

holds for some constant $c$ independent of $\tau$ and $\Gamma$. Recall that $\tau$ is proportional to $\beta$. Since $Z_{q}^{t}(\Lambda, u)=1$ if $\ell(\Lambda)=0$, the bound in the lemma holds for $\ell(\operatorname{Int} \Gamma)=0$, and sufficiently large $\tau$. 
Notice that by the definition of $Z^{t}(\operatorname{Int} \Gamma, u)$ in (2.14),

$$
\begin{aligned}
\frac{d}{d u} Z^{t}(\operatorname{Int} \Gamma, u)= & \sum_{\partial \in \mathscr{E}(\operatorname{Int} \Gamma)} \sum_{\Gamma^{\prime} \in \hat{\partial} \partial} \int d \omega\left[-\Psi^{t}\left(\Gamma^{\prime}\right)\right. \\
& \left.+\left(\left\{\frac{d}{d u} Z^{t}\left(\operatorname{Int} \Gamma^{\prime}, u\right)\right\} / Z^{t}\left(\operatorname{Int} \Gamma^{\prime}, u\right)\right)\right] \\
& \cdot e^{-H_{u}^{t}(\hat{\partial} ; \omega)} \prod_{\Gamma^{\prime \prime} \in \hat{\partial}} Z^{t}\left(\operatorname{Int} \Gamma^{\prime \prime}, u\right) / Z\left(\operatorname{Int} \Gamma^{\prime \prime}\right) \\
= & \sum_{\Gamma^{\prime} \in \mathscr{E}(\operatorname{Int} \Gamma)}\left[-\Psi^{t}\left(\Gamma^{\prime}\right)+\left(\left\{\frac{d}{d u} Z^{t}\left(\operatorname{Int} \Gamma^{\prime}, u\right)\right\} / Z^{t}\left(\operatorname{Int} \Gamma^{\prime}, u\right)\right)\right] \\
& \cdot \rho_{\operatorname{Int} \Gamma}^{t}\left(\Gamma^{\prime}, u\right) Z^{t}(\operatorname{Int} \Gamma, u) .
\end{aligned}
$$

Here we have summed over $\partial-\Gamma^{\prime}$ to get the second equality. Thus we get the bound

$$
\begin{aligned}
\left|\left\{\frac{d}{d u} Z^{t}(\operatorname{Int} \Gamma, u)\right\} / Z^{t}(\operatorname{Int} \Gamma, u)\right| \leqq & \sum_{\Gamma^{\prime} \in \mathscr{G}(\operatorname{In} T)}\left\{\left|\Psi^{t}\left(\Gamma^{\prime}\right)\right|\right. \\
& \left.+\left|\left\{\frac{d}{d u} Z^{t}\left(\operatorname{Int} \Gamma^{\prime}, u\right)\right\} / Z^{t}\left(\operatorname{Int} \Gamma^{\prime}, u\right)\right|\right\} \rho_{\text {Int } \Gamma^{t}\left(\Gamma^{\prime}, u\right) .}
\end{aligned}
$$

We use (3.3), the bound in Theorem 2.2(a), i.e.,

$$
\rho_{\text {Int } \Gamma}^{t}\left(\Gamma^{\prime}, u\right) \leqq e^{-\tau \mid \Gamma^{\prime} / / 6}
$$

uniformly in $u \geqq 1$, and the fact that by Lemma 4.2 .1 of I

$$
c^{\prime} \sum_{\Gamma^{\prime} \in \mathscr{C}(\ln T)} \tau\left|V\left(\Gamma^{\prime}\right)\right|^{2} e^{-\tau \mid \Gamma^{\prime} / 6} \leqq|\operatorname{Int} \Gamma|
$$

for sufficiently large $\tau$ to obtain from (3.5) that

$$
\begin{aligned}
& \left|\left\{\frac{d}{d u} Z^{t}(\operatorname{Int} \Gamma, u)\right\} / Z^{t}(\operatorname{Int} \Gamma, u)\right| \\
& \quad \leqq|\operatorname{Int} \Gamma|+\sum_{\Gamma^{\prime} \in \mathscr{G}(\operatorname{Int} \Gamma)}\left|\left\{\frac{d}{d u} Z^{t}\left(\operatorname{Int} \Gamma^{\prime}, u\right)\right\} / Z^{t}\left(\operatorname{Int} \Gamma^{\prime}, u\right)\right| e^{-\tau \mid \Gamma^{\prime} / 6} .
\end{aligned}
$$

Let $\ell(\operatorname{Int} \Gamma)=n$, then $\ell\left(\operatorname{Int} \Gamma^{\prime}\right) \leqq n-1$ for any $\Gamma^{\prime} \in \mathscr{C}(\operatorname{Int} \Gamma)$. Now the lemma follows from (3.7) and the induction on the level of $\Gamma^{\prime}$. Also one may show the lemma by the following argument: Iterating (3.7) $n$-times and using (3.6) it is easy to show that (3.7) is bounded by $n|\operatorname{Int} \Gamma| \leqq|V(\Gamma)|^{2}$. This completes the proof of the lemma.

We are now ready to prove Proposition 3.1.

Proof of Proposition 3.1. For a notational simplification, write that

$$
Q_{q}^{t}(\Gamma, u)=-\Psi^{t}(\Gamma)+\left(\left\{\frac{d}{d u} Z_{q}^{t}(\operatorname{Int} \Gamma, u)\right\} / Z_{q}^{t}(\operatorname{Int} \Gamma, u)\right)
$$


Then from (3.1), (2.18) and (3.4) it follows that

$$
s_{\Lambda, q}=-|\Lambda|^{-1} \int_{1}^{\infty} d u\left\{\frac{d}{d u} Z_{q}^{t}(\Lambda, u)\right\} Z_{q}^{t}(\Lambda, u)^{-1}=-|\Lambda| \int_{1}^{\infty} d u \sum_{\Gamma \in \mathscr{G}(\Lambda)} Q_{q}^{t}(\Gamma, u) \rho_{\Lambda}^{t}(\Gamma, u) .
$$

Thus it is not hard to recognize that

$$
s_{q}=-\int_{1}^{\infty} d u \frac{1}{|\Gamma|} \sum_{\substack{\Gamma \cdot \operatorname{Supp} \Gamma \\ S_{q}}} Q_{q}^{t}(\Gamma, u) \rho^{t}(\Gamma, u)
$$

By Theorem 2.2(a) and Lemma 3.3, $s_{q} \rightarrow 0$ as $\tau \rightarrow \infty$.

In order to show the bound in the proposition, define

Then

$$
s_{\Lambda, q}^{\prime} \equiv-|\Lambda|^{-1} \int_{1}^{\infty} d u \sum_{\Gamma \in \mathscr{C}(\Lambda)} Q_{q}^{t}(\Gamma, u) \rho^{t}(\Gamma, u) .
$$

$$
\begin{aligned}
\left|\log Z^{t}(\Lambda)-s_{q}\right| \Lambda|| & =\left|s_{\Lambda, q}\right| \Lambda\left|-s_{q}\right| \Lambda|| \\
& \leqq\left|s_{\Lambda, q}-s_{\Lambda, q}^{\prime}\right||\Lambda|+\left|s_{\Lambda, q}^{\prime}-s_{q}\right||\Lambda| .
\end{aligned}
$$

Using (3.9), (3.11), (3.8), Lemma 3.3, Theorem 2.2(b) and Assumption 1.1 we obtain

$$
\begin{aligned}
\left|s_{\Lambda, q}-s_{\Lambda, q}^{\prime}\right| \Lambda \mid & \leqq \int_{1}^{\infty} d u \sum_{\Gamma \in \mathscr{G}(\Lambda)}\left|Q_{q}^{t}(\Gamma, u)\right|\left|\rho_{\Lambda}^{t}(\Gamma, u)-\rho^{t}(\Gamma, u)\right| \\
& \leqq \int_{1}^{\infty} d u \sum_{\Gamma \in \mathscr{G}(\Lambda)} \tau|V(\Gamma)|^{2} e^{-u \tau|\Gamma| / 6} e^{-\delta\left(\Gamma, \Lambda^{c}\right)} \\
& \leqq \sum_{\Gamma \in \mathscr{C}(\Lambda)} 6 \tau|V(\Gamma)|^{2} e^{-\tau|\Gamma| / 6} e^{-\delta\left(\Gamma, \Lambda^{\alpha}\right)} \\
& \leqq c(\tau) \sum_{x \in \Lambda} e^{-\delta\left(x, \Lambda^{c}\right)} \leqq \varepsilon_{1}(\tau)|\operatorname{bd}(\Lambda)|,
\end{aligned}
$$

where $\varepsilon_{1}(\tau) \rightarrow 0$ as $\tau \rightarrow \infty$. Here we have used Lemma 4.2.1 of I to get the third inequality.

For any $B \subset \mathbb{Z}^{v}$, let $[B]=\{\Gamma \in \mathscr{C}$ : $\operatorname{dist}(\Gamma, B) \leqq 1\}$. Then from (3.10), (3.11), Lemma 3.3 and Theorem 2.2(a) it follows that

$$
\begin{aligned}
\left|s_{\Lambda, q}^{\prime}-s_{q}\right||\Lambda| & \leqq \sum_{\Gamma \in[\operatorname{bd}(\Lambda)]} \int_{1}^{\infty} d u\left|Q_{q}^{t}(\Gamma, u)\right| \rho^{t}(\Gamma, u) \\
& \leqq \sum_{\Gamma \in[\operatorname{bd}(\Lambda)]} 6 \tau^{2}|V(\Gamma)|^{2} e^{-\tau(\Gamma) / 6} \leqq \varepsilon_{2}(\tau)|\operatorname{bd}(\Lambda)|,
\end{aligned}
$$

where $\varepsilon_{2}(\tau) \rightarrow 0$ as $\tau \rightarrow \infty$. The bound in the proposition follows from (3.13) and (3.14).

We next prove Proposition 3.2.

Proof of Proposition 3.2. Denote the contour Hamiltonian corresponding to the Hamiltonian $H_{\lambda}=H_{0}+\lambda \tilde{H}$ by

$$
H_{\lambda}(\partial ; \omega)=\sum_{\Gamma \in \hat{\partial}} \Phi_{1, \lambda}(\Gamma, \omega)+\sum_{\Gamma, \Gamma^{\prime} \in \hat{C}} \Phi_{2, \lambda}\left(\Gamma, \Gamma^{\prime} ; \omega\right),
$$




$$
\begin{aligned}
\Phi_{1, \lambda}(\Gamma ; \omega) & =\Phi_{1}(\Gamma ; \omega)+\lambda \tilde{\Phi}_{1}(\Gamma, \omega), \\
\Phi_{2, \lambda}\left(\Gamma, \Gamma^{\prime} ; \omega\right) & =\Phi_{2}\left(\Gamma, \Gamma^{\prime} ; \omega\right)+\lambda \tilde{\Phi}_{2}\left(\Gamma, \Gamma^{\prime} ; \omega\right),
\end{aligned}
$$

where $\tilde{\Phi}_{1}$ and $\tilde{\Phi}_{2}$ are the one body and two body contour interactions corresponding to $\tilde{H}$. Let $Z_{q, \lambda}(\Lambda), Z_{q, \lambda}(\Gamma)$ and $\Psi_{\lambda}(\Gamma)$ be the partition function, the crystal partition function and the contour functional corresponding to $H_{\lambda}$ respectively. We also denote the corresponding truncated contour functional, partition function and correlation functions by $\Psi_{\lambda}^{t}(\Gamma), Z_{q, \lambda}^{t}(\lambda)$ and $\rho_{\Lambda \cdot q, \lambda}^{t}(\partial)$ respectively. Then the refined truncated contour correlation functions for $H_{\lambda}$ are defined by

$$
\begin{aligned}
\rho_{\Lambda, q, \lambda}^{t}(\partial ; \omega)= & \frac{1}{Z_{q, \lambda}^{t}(\Lambda)} \sum_{\hat{o}^{\prime} \in \mathscr{E}(\Lambda \backslash V(\hat{\partial}))} \int_{\hat{o}^{\prime}} d \omega e^{-H_{\lambda}^{t}\left(\partial \cup \hat{o}^{\prime}, \omega\right)} \\
& \cdot \prod_{\Gamma \in \hat{\partial} \cup \hat{\sigma}^{\prime}}\left[Z_{q, \lambda}^{t}(\operatorname{Int} \Gamma) / Z_{q, \lambda}(\operatorname{Int} \Gamma)\right],
\end{aligned}
$$

and then it follows that

$$
\rho_{\Lambda, q, \lambda}^{t}(\partial)=\int_{\hat{\delta}} d \omega \rho_{\Lambda, q, \lambda}^{t}(\partial, \omega) .
$$

As a consequence of Proposition 2.1, the infinite volume limit $\rho_{q, \lambda}^{t}(\partial ; \omega)$ of $\rho_{\Lambda, q, \lambda}^{t}(\partial ; \omega)$ exists for each $\partial \in \mathscr{E}\left(\mathbb{Z}^{v}\right)$ and $\omega \in \Omega(\partial)$.

Next, we denote

$$
s_{\Lambda, q}(\lambda)=|\Lambda|^{-1} \log Z_{q, \lambda}^{t}(\Lambda) .
$$

For typological convenience we simply write the one-side derivative $(d / d \lambda( \pm)) f(\lambda)$ by $(d / d \lambda) f(\lambda)$ in the following proof. A direct calculation yields

$$
\begin{aligned}
& \frac{d}{d \lambda}\left\{Z_{q, \lambda}^{t}(\Lambda) / Z_{q, \lambda}(\Lambda)\right\} \\
&=\left[\left\{\left(\frac{d}{d \lambda} Z_{q, \lambda}^{t}(\Lambda)\right) / Z_{q, \lambda}^{t}(\Lambda)\right\}-\left\{\left(\frac{d}{d \lambda} Z_{q, \lambda}(\Lambda)\right) / Z_{q, \lambda}(\Lambda)\right\}\right] \\
& \cdot\left\{Z_{q, \lambda}^{t}(\Lambda) / Z_{q, \lambda}(\Lambda)\right\} .
\end{aligned}
$$

Using (2.14), (3.16) and (3.19) it can be shown that

where

$$
\begin{aligned}
\left(\frac{d}{d \lambda} Z_{q, \lambda}^{t}(\Lambda)\right) / Z_{q, \lambda}^{t}(\Lambda)= & -\sum_{\left\{\Gamma, \Gamma^{\prime}\right\} \in \delta(\Lambda)} \int_{\Gamma \cup \Gamma^{\prime}} d \omega \tilde{\Phi}_{2}\left(\Gamma, \Gamma^{\prime} ; \omega\right) \rho_{\Lambda, q, \lambda}^{t}\left(\Gamma \cup \Gamma^{\prime} ; \omega\right) \\
& +\sum_{\Gamma \in \mathscr{C}(\Lambda) \Gamma} \int_{\Gamma} d \omega B_{q, \lambda}^{t}(\Gamma ; \omega) \rho_{\Lambda, q, \lambda}^{t}(\Gamma ; \omega),
\end{aligned}
$$

$$
\begin{aligned}
B_{q, \lambda}^{t}(\Gamma ; \omega) \equiv & -\frac{d}{d \lambda} \Phi_{1, \lambda}^{t}(\Gamma ; \omega)+\left\{\left(\frac{d}{d \lambda} Z_{q, \lambda}^{t}(\operatorname{Int} \Gamma)\right) / Z_{q, \lambda}^{t}(\operatorname{Int} \Gamma)\right\} \\
& +\left\{\left(\frac{d}{d \lambda} Z_{q, \lambda}(\operatorname{Int} \Gamma)\right) / Z_{q, \lambda}(\operatorname{Int} \Gamma)\right\}
\end{aligned}
$$

and

$$
\Phi_{1, \lambda}^{t}(\Gamma ; \omega)=\Phi_{1, \lambda}(\Gamma ; \omega)+\Psi_{\lambda}^{t}(\Gamma)-\Psi_{\lambda}(\Gamma)
$$


Since the cluster expansion converges absolutely, and uniformly in $\Lambda$, it is easy to show that by (3.20),

$$
\begin{aligned}
\frac{d}{d \lambda} s_{q}(\lambda)= & \lim _{\Lambda \rightarrow \mathbb{Z}^{v}}|\Lambda|^{-1}\left(\frac{d}{d \lambda} Z_{q, \lambda}^{t}(\Lambda)\right) / Z_{q, \lambda}^{t}(\Lambda) \\
= & -\sum_{\substack{\Gamma: \\
0 \in \operatorname{Supp} \Gamma}} \frac{1}{|\Gamma|} \sum_{\Gamma^{\prime} \in \mathcal{E}\left(\mathscr{X}^{v} \backslash V(\Gamma)\right)} \int_{\Gamma \cup \Gamma^{\prime}} d \omega \tilde{\Phi}_{2}\left(\Gamma, \Gamma^{\prime} ; \omega\right) \rho_{q, \lambda}^{t}\left(\Gamma \cup \Gamma^{\prime} ; \omega\right) \\
& +\sum_{\substack{\Gamma \cdot \\
0 \in \operatorname{Supp} \Gamma}} \frac{1}{|\Gamma|} \int_{\Gamma} d \omega B_{q, \lambda}^{t}(\Gamma ; \omega) \rho_{q, \lambda}^{t}(\Gamma ; \omega) .
\end{aligned}
$$

Using the definition of $Z_{q, \lambda}(\Lambda)$ and Lemma 4.2.1 of Part I, one may check that

$$
\begin{aligned}
& \left|\left(\frac{d}{d \lambda} Z_{q, \lambda}(\operatorname{Int} \Gamma)\right) / Z_{q, \lambda}(\operatorname{Int} \Gamma)\right| \leqq c_{1} \tau|V(\Gamma)|, \\
& \sum_{\Gamma^{\prime} \in \mathscr{G}(\Lambda \backslash V(\Gamma))}\left|\tilde{\Phi}_{2}\left(\Gamma, \Gamma^{\prime} ; \omega\right)\right| \leqq c_{2} \tau|V(\Gamma)|, \quad\left|\tilde{\Phi}_{1}(\Gamma ; \omega)\right| \leqq c_{3} \tau|V(\Gamma)|
\end{aligned}
$$

for some universal constant $c_{1}, c_{2}$ and $c_{3}$. Using the facts that

$$
\left|\frac{d}{d \lambda} \log Z_{q, \lambda}(\Gamma)\right| \leqq c_{4}|V(\Gamma)|, \quad\left|\frac{d}{d \lambda} \log Z_{q, \lambda}(\operatorname{Int} \Gamma)\right| \leqq c_{5}|V(\Gamma)|,
$$

and (3.22), and the third inequality in (3.24), it can be shown that

$$
\left|\frac{d}{d \lambda} \Phi_{1, \lambda}^{t}(\Gamma, \omega)\right| \leqq c \tau|V(\Gamma)|
$$

for some constant $c$ uniformly in $\omega \in \Omega(\Gamma)$. Thus, by (3.20), (3.21), (3.24) and (3.25) we get the bound

$$
\begin{aligned}
& \left|\left(\frac{d}{d \lambda} Z_{q, \hat{\lambda}}^{t}(\Lambda)\right) / Z_{q, \lambda}^{t}(\Lambda)\right| \\
& \quad \leqq \sum_{\Gamma \in \mathscr{C}(\Lambda)}\left[c \tau|V(\Gamma)|+\left|\left(\frac{d}{d \lambda} Z_{q, \lambda}^{t}(\operatorname{Int} \Gamma)\right) / Z_{q, \lambda}^{t}(\operatorname{Int} \Gamma)\right|\right] \rho_{\Lambda, q, \lambda}^{t}(\Gamma) .
\end{aligned}
$$

We now use the induction procedure on the level of $\Lambda$ similar to that used in the proof of Lemma 3.3 to conclude that

$$
\left|\frac{d}{d \lambda} Z_{q, \lambda}^{t}(\operatorname{Int} \Gamma) / Z_{q, \lambda}^{t}(\operatorname{Int} \Gamma)\right| \leqq \tau|\operatorname{Int} \Gamma|^{2},
$$

and so by (3.25), (3.26) and (3.24),

$$
\left|B_{q, \lambda}^{t}(\Gamma ; \omega)\right| \leqq c \tau|V(\Gamma)|^{2}
$$

Now the bound in the proposition follows from (3.23), (3.24), (3.27) and Lemma 4.2.1 of Part I together with the fact that $\rho_{q, \lambda}^{t}(\partial) \leqq \exp (-\tau|\partial| / 6)$. This completes the proof of the proposition. 


\section{Bounds on Partition Functions and Proof of the Main Theorem}

In this section we establish upper and lower bounds on the partition function and also obtain a sufficient condition on ground states $\omega_{q}, q \in Q$, to be stable. Then the proof of the main theorem will be followed from the bounds, the Lipschitz continuity for $s_{q}$ (Proposition 3.2) and the method used in Zahradnik [6].

Let $e_{q}$ be the specific energy defined in (1.6) and let $s_{q}$ be the free energy for the truncated model. Write that for any $q \in Q$,

$$
h_{q}=e_{q}-s_{q}, \quad h_{0}=\min \left\{h_{q}\right\}, \quad a_{q}=h_{q}-h_{0}, \quad a=\min \left\{a_{q}: a_{q} \neq 0\right\} .
$$

The following is the result analogous to Theorem 1.7 of Zahradnik [6].

Theorem 4.1. Nonstable contours satisfy the inequality

$$
a_{q}\left|\operatorname{Int} \Gamma^{q}\right| \geqq \tau\left|\Gamma^{q}\right| / 4 \text {. }
$$

Thus, if $a_{q}=0$, then $q \in Q$ is stable. That is, all contours $\Gamma \in \mathscr{C}_{q}$ are stable and $\Psi^{t}(\Gamma)$ $=\Psi(\Gamma)$ for all $\Gamma \in \mathscr{E}_{q}$. Furthermore there is a universal constant $c(\tau)$ such that for each finite $\Lambda \subset \mathbb{Z}^{v}$ the following estimates hold:

$$
\begin{aligned}
& \exp \left(-e_{q}|\Lambda|\right) Z_{q}(\Lambda) \geqq \exp \left[-h_{q}|\Lambda|-c|\operatorname{bd}(\Lambda)|\right], \\
& \exp \left(-e_{q}|\Lambda|\right) Z_{q}(\Lambda) \leqq \exp \left[-h_{0}|\Lambda|+c|\operatorname{bd}(\Lambda)|\right] .
\end{aligned}
$$

The constant $c=c(\tau)$ can be chosen such that $c(\tau) \rightarrow 0$ as $\tau \rightarrow \infty$.

We postpone the proof of the above result to the end of this section.

We use Theorem 4.1 and Proposition 3.2 to prove Theorem 1.3.

Proof of Theorem 1.3. We remark that contrary to our case, the factor $\exp \left(-e_{q}|\Lambda|\right)$ was included in Zahradnik's definition of the partition function [6]. Using the Lipschitz continuity of the free energy (Proposition 3.2), Theorem 4.1 and the method same as that used in Sect. 1.10 of [6] one can construct the homeomorphism in Theorem 1.3. The stability of $q$ with $h^{0}(\lambda)_{q}=0$, where $h^{0}(\lambda)_{q} \equiv a_{q}(\lambda)$, follows from the bound in (4.2). Thus for each $q \in Q(\lambda)$ the infinite volume limit of the refined contour correlation function $\rho_{q}(\partial ; \omega)$ exists for any $\partial \in \mathscr{E}_{q}$ and $\omega \in \Omega(\partial)$. Using the correlation functions one can construct a Gibbs measure $\rho_{q}$ on $\Omega^{\mathbb{Z}^{v}}$ (e.g. see [5]). As a consequence of the cluster property it follows that the Gibbs state $\rho_{q}$ is pure.

In order to show Theorem 4.1, we need the following result:

Proposition 4.2. Let the conditions in Assumption 1.1 and Assumption 1.2 be satisfied. For a given external q-contour $\Gamma=\left(M, \omega_{M}\right)$, let Int $\Gamma=\bigcup_{q^{\prime} \in Q} \operatorname{Int}_{q^{\prime}} \Gamma$. Then the bound

$$
\begin{aligned}
\left\{Z_{q}(\Gamma) / Z_{q}(\operatorname{Int} \Gamma)\right\} \leqq & \left\{\prod_{q^{\prime} \in Q}\left[Z_{q^{\prime}}\left(\operatorname{Int}_{q^{\prime}} \Gamma\right) / Z_{q}\left(\operatorname{Int}_{q^{\prime}} \Gamma\right)\right]\right\} \\
& \cdot \exp \left\{-\frac{2 \tau}{3}|\Gamma|-\sum_{q^{\prime} \in Q}\left(e_{q^{\prime}}-e_{q}\right)\left|\operatorname{Int}_{q^{\prime}} \Gamma\right|\right\}
\end{aligned}
$$

holds for any $\Gamma \in \mathscr{C}_{q}$.

Since the proof of the proposition is somewhat lengthy, we will produce the proof in the appendix. 
In the rest of this section we prove Theorem 4.1 by employing Zahradnik's idea in Sect. 1.8 of [6] together with our previous results.

Proof of Theorem 4.1. As in [6], we will proceed by induction over the levels of $\Lambda$ and $\Gamma$.

(a) Proof of (4.3). Because $Z_{q}(\Lambda) \geqq Z_{q}^{t}(\Lambda)$ by (2.16) and

$$
Z_{q}^{t}(\Lambda) \geqq \exp \left(s_{q}|\Lambda|-c|\mathrm{bd}(\Lambda)|\right)
$$

by Proposition 4.2, we get the desired relation in (4.3).

(b) Proof of (4.2). We use the induction argument for (4.4). Both (4.2) and (4.4) are trivial if both $\Gamma$ and $\Lambda$ have zero levels. Nonstability of $\Gamma$ means, by (1.7),

$$
Z_{q}(\Gamma) / Z_{q}(\operatorname{Int} \Gamma) \geqq \exp (-\tau|\Gamma| / 3) .
$$

Since $\ell($ Int $\Gamma)<\ell(\Gamma)$, we use the induction assumption for (4.4) to obtain

$$
\exp \left(-e_{q^{\prime}}\left|\operatorname{Int}_{q^{\prime}} \Gamma\right|\right) Z_{q^{\prime}}\left(\operatorname{Int}_{q^{\prime}} \Gamma\right) \leqq \exp \left(-h_{0}\left|\operatorname{Int}_{q^{\prime}} \Gamma\right|+c\left|\operatorname{bd}\left(\operatorname{Int}_{q^{\prime}} \Gamma\right)\right|\right) .
$$

From Proposition 4.2, (4.3) and the above bound it follows that

$$
Z_{q}(\Gamma) / Z_{q}(\operatorname{Int} \Gamma) \leqq \exp (-\tau|\Gamma| / 3) \exp \left(-h_{0}|\operatorname{Int} \Gamma|+h_{q}|\operatorname{Int} \Gamma|+2 c \mid \text { bd }(\operatorname{Int} \Gamma) \mid\right) \text {. }
$$

Combining the inequalities in (4.5) and (4.6) we get

$$
0 \leqq-(\tau|\Gamma| / 3)+a_{q}|\operatorname{Int} \Gamma|+2 c \mid \text { bd }(\operatorname{Int} \Gamma) \mid \text {. }
$$

This proves (4.3) for sufficiently large $\tau$.

(c) Proof of (4.4). Notice that, if all $q$-contours are stable, then $Z_{q}(\Lambda)=Z_{q}^{t}(\Lambda)$, and so (4.4) follows from Proposition 3.1. Thus we need some method of handling the unstable contours.

We use the method of Zahradnik [6]. We say that a contour $\Gamma^{q}$ is small if it is stable and there is no unstable contour $\widetilde{\Gamma}^{q}$ such that supp $\widetilde{\Gamma}^{q} \subset \operatorname{Int} \Gamma^{q}$. Say that $\Gamma^{q}$ is large if it is not small.

Given any configuration $\omega(x)=q$ for all $x \in\left(\Lambda_{s}\right)^{c}$, one may write the external boundary of $\omega$ by ext $(\partial \omega)=\partial \cup \partial_{\ell}$, where $\partial_{\ell}=\left\{\Gamma_{t, 1}, \ldots, \Gamma_{t, n}\right\}$ is the collection of all external large contours of $\omega$ and any $\Gamma \in \partial$ is a small external $q$-contour of $\omega$. Denote $L \mathscr{E}_{q}(\Lambda)$ (respectively $S \mathscr{E}_{q}(\Lambda)$ ) the family of external $q$-contour systems consisting of large (small $q$-contours). Then we get the relation

$$
Z_{q}(\Lambda)=\sum_{\hat{c} \in L \mathscr{E}(\Lambda)} \sum_{\hat{c}^{\prime} \in S \mathscr{E}(\Lambda \backslash V(\hat{c}))} \int_{\hat{c} \cup \hat{c}^{\prime}} d \omega e^{-H\left(\hat{c} \cup \hat{o}^{\prime} ; \omega\right)} .
$$

Note that by the definition of small contours, any $\Gamma \in \mathscr{C}\left(\operatorname{Int} \partial^{\prime}\right), \partial^{\prime} \in S \mathscr{E}(\Lambda)$, is small contour. Write

$$
\tilde{Z}_{q}(\Lambda) \equiv \sum_{\hat{c} \in S \mathscr{E}(\Lambda)} \int_{\hat{\partial}} d \omega e^{-H(\hat{c} ; \omega)}
$$

and let $\tilde{s}_{q}$ be the free energy corresponding to $\tilde{Z}_{q}(\Lambda)$. Since $Z_{q}^{t}(\operatorname{Int} \Gamma)=Z_{q}(\operatorname{Int} \Gamma)$ for any small $\Gamma, \tilde{Z}_{q}(\Lambda)$ is equivalent to the truncated partition function $Z_{q}^{t}(\Lambda)$ with a contour functional

$$
\begin{array}{ll}
\tilde{\Psi}(\Gamma)=\Psi(\Gamma) & \text { if } \Gamma \text { is small, } \\
\widetilde{\Psi}(\Gamma)=\infty & \text { if } \Gamma \text { is large. }
\end{array}
$$


Obviously, $\tilde{s}_{q} \leqq s_{q}$. Using Proposition 3.1 (and a suitable modification of the method used in the proof of Proposition 3.1) we get the bound

$$
\exp \left(-e_{q}|\Lambda|\right) \tilde{Z}_{q}(\Lambda) \leqq \exp \left(-\tilde{h}_{q}|\Lambda|+c|\operatorname{bd}(\Lambda)|\right),
$$

where $\tilde{h}_{q}=e_{q}-\tilde{s}_{q}$. By the induction assumption for (4.4) it follows that for any $\Gamma \in \partial$, $\partial \in L \mathscr{E}(\Lambda)$, the bound

$$
\exp \left(-e_{q^{\prime}}\left|\operatorname{Int}_{q^{\prime}} \Gamma\right|\right) Z_{q}\left(\operatorname{Int}_{q^{\prime}} \Gamma\right) \leqq \exp \left(-h_{0}\left|\operatorname{Int}_{q^{\prime}} \Gamma\right|+c\left|\operatorname{bd}\left(\operatorname{Int}_{q^{\prime}} \Gamma\right)\right|\right)
$$

holds.

Next, writing $H\left(\partial \cup \partial^{\prime} ; \omega\right)=H(\partial ; \omega)+H\left(\partial^{\prime} ; \omega\right)+\Phi_{2}\left(\partial, \partial^{\prime} ; \omega\right)$ and $H(\partial ; \omega)=$ $\sum_{\Gamma \in \partial} \Phi_{1}(\Gamma ; \omega)+\sum_{\Gamma, \Gamma^{\prime} \in \hat{o}} \Phi_{2}\left(\Gamma, \Gamma^{\prime} ; \omega\right)$, and using Proposition 4.2.2(a) of I, it follows from (4.7) that

$$
Z_{q}(\Lambda) \leqq \sum_{\partial \in L \mathscr{E}(\Lambda)} \int_{\hat{c}} d \omega \tilde{Z}_{q}(\Lambda \backslash V(\partial)) \exp \left(-\sum_{\Gamma \in \hat{\partial}} \Phi_{1}(\Gamma ; \omega)\right) \exp (-\tau|\partial| / 24) .
$$

Using (A.34) in the appendix into (4.11) we obtain the bound

$$
\begin{aligned}
Z_{q}(\Lambda) \leqq & \sum_{\hat{o} \in L \delta(\Lambda)} \tilde{Z}_{q}(\Lambda \backslash V(\partial))\left\{\prod_{\Gamma \in \hat{c}}\left[\prod_{q^{\prime} \in Q} Z_{q^{\prime}}\left(\operatorname{Int}_{q^{\prime}} \Gamma\right) \exp \left(-\left(e_{q^{\prime}}-e_{q}\right)\left|\operatorname{Int}_{q^{\prime}} \Gamma\right|\right)\right]\right. \\
& \cdot \exp (-\Phi(\Gamma)+\tau|\Gamma| / 8)\} .
\end{aligned}
$$

Denote $\tilde{a}_{q}=\tilde{h}_{q}-h_{0}$, and use (4.9), (4.10), and (4.12) to obtain the bound

$$
\begin{aligned}
\exp \left(-e_{q}|\Lambda|\right) Z_{q}(\Lambda) \leqq & \exp \left(-h_{0}|\Lambda|\right) \sum_{\hat{\partial} \in L \mathscr{E}(\Lambda)} \exp \left(-\tilde{a}_{q}|\Lambda \backslash V(\partial)|\right) \\
& \cdot\left\{\prod_{\Gamma \in \hat{o}} \exp \left[-\Phi(\Gamma)+\left(h_{0}-e_{q}\right)|\Gamma|\right] \exp (\tau|\Gamma| / 8)\right\} \\
& \cdot\left\{\exp \left[c\left(2 \sum_{\Gamma \in \hat{o}}|\operatorname{bd}(\operatorname{Int} \Gamma)|+c|\operatorname{bd}(\Lambda \backslash V(\partial))|\right]\right\} .\right.
\end{aligned}
$$

The above is the bound the same as that of (1.39) in [6] except for the factor $\exp (\tau|\Gamma| / 8)$ which can be controlled by $\exp (-\Phi(\Gamma))$ and the Peierls condition. Note that $\tilde{a}_{q} \geqq a_{q}$ and $a_{q} \geqq a$ if there is a unstable contour $\Gamma^{q}$. Thus using the method by Zahradnik (page 567 of [6]) we proved the bound (4.4) completely.

\section{An Application. Potts Model with Infinite Range Interactions}

In order to give an example of how our results can be applied, let us consider Potts models with infinite range interactions. Let $\Omega=\{1,2, \ldots, r\}$. The unperturbed Hamiltonian $H_{0}$ is given by

$$
H_{0}=-\beta \sum_{\{x, y\}} K(x-y) \tilde{\delta}(\omega(x), \omega(y)),
$$

where $\tilde{\delta}(p, q)=1$ if $p=q$ and $\tilde{\delta}(p, q)=0$ if $p \neq q$. Here we have used the symbol $\tilde{\delta}$ instead of $\delta$ to avoid confusion with the metric $\delta$ in Assumption 1.1. We impose the following condition on $K(x-y)$.

Assumption 5.1. [Regularity condition] The function $K: \mathbb{Z}^{v} \rightarrow \mathbb{R}$ is symmetric with 
respect to $x \rightarrow-x$ and satisfies the bound

$$
2|K(x-y)| \leqq c\|x-y\|^{-(\lambda+\varepsilon)}
$$

for some constants $\lambda>2 v+1, \varepsilon>v$ and $c>0$.

For the Hamiltonian (5.1) it is easy to check that any $q, q \in \Omega$, is a ground state, and so we choose $Q=\Omega$. The specific energy is given by

$$
e_{q}\left(H_{0}\right)=-\frac{1}{2} \beta \sum_{x \in \mathbb{Z}^{v}} K(x)
$$

for any $q \in Q$. In order to split the degeneracy of the ground states we introduce the external fields

$$
H_{q}=\beta \sum_{x \in \mathbb{Z}^{v}} \tilde{\delta}(\omega(x), q), \quad q \in Q
$$

It is easy to see that the perturbation

$$
H_{\lambda}=H_{0}+\sum_{i=1}^{r-1} \lambda_{i} H_{i}
$$

splits the degeneracy of the ground states.

We recall the definition of $\Phi(\Gamma)$ in (1.2). For the Hamiltonian $H_{\lambda}$ in (5.4) the contour functional $\Phi(\Gamma)$ is given by

$$
\Phi(\Gamma)=\Phi_{0}(\Gamma)+\tilde{\Phi}_{\lambda}(\Gamma)
$$

where for any $q$-contour $\Gamma=\left(M, \omega_{M}\right)$,

$$
\begin{aligned}
& \Phi_{0}(\Gamma)=-\beta \sum_{\substack{\{x, y\} \cap M \neq \phi \\
|x-y| \leqq s}} \frac{1}{2}|\{x, y\} \cap M|\{K(x-y) \tilde{\delta}(\omega(x), \omega(y))-K(x-y)\}, \\
& \tilde{\Phi}_{\lambda}(\Gamma)=\beta \sum_{q^{\prime}=1}^{r-1} \sum_{x \in M} \lambda_{q^{\prime}}\left\{\tilde{\delta}\left(\omega(x), q^{\prime}\right)-\tilde{\delta}\left(q, q^{\prime}\right)\right\} .
\end{aligned}
$$

Notice that $\tilde{\delta}(\omega(x), \omega(y))=1$ if $x \in M, y \in M^{c}$ and $\|x-y\| \leqq s$. Thus $\Phi_{0}(\Gamma)$ can be written as

$$
\Phi_{0}(\Gamma)=\sum_{x \in M}\left\{-\frac{\beta}{2} \sum_{\substack{y \in \mathbb{Z}^{v} \\\|x-y\| \leqq}}[K(x-y) \tilde{\delta}(\omega(x), \omega(y))-K(x-y)]\right\} .
$$

In order to satisfy the Peierls condition one needs to impose additional restrictions on the function $K(x-y)$.

Let us first consider the case for ferromagnetic interactions, i.e., $K(x-y) \geqq 0$ for any $x, y \in \mathbb{Z}^{v}$. For any $x \in \mathbb{Z}^{v}$, let

$$
|x|=\left(\sum_{i=1}^{v}\left|x_{i}\right|^{2}\right)^{1 / 2}
$$

and let

$$
K_{0}=\min \{K(x):|x|=1\} .
$$

We then have the following result: 
Theorem 5.2. Let $K(x-y)$ satisfy the bound in Assumption 5.1. Assume that $K(x-y) \geqq 0$ for all $x, y \in \mathbb{Z}^{v}$ and $K_{0}>0$. Then one can choose the parameter such that the results in Theorem 1.3 hold for sufficiently large $\beta$.

Proof. By the definition of contours one may check that for any contour $\Gamma=\left(M, \omega_{M}\right)$ there are at least $(2 s+1)^{-v}|\Gamma|$ number of distinct neighboring pairs $\langle x, y\rangle$ in support $\Gamma$ such that $\omega(x) \neq \omega(y)$. Thus by (5.7)

$$
\Phi_{0}(\Gamma) \geqq(2 s+1)^{-v} \beta K_{0}|\Gamma| \text {. }
$$

On the other hand it follows from (5.6) that for $|\lambda| \equiv \sum_{i=1}^{n-1}\left|\lambda_{q_{l}}\right|$,

$$
\left|\tilde{\Phi}_{\lambda}(\Gamma)\right| \leqq|\lambda| \beta|\Gamma|
$$

Thus from (5.10) and (5.11) we get that

$$
\Phi(\Gamma) \geqq\left((2 s+1)^{-v} K_{0}-|\lambda|\right) \beta|\Gamma| .
$$

Let $|\lambda| \leqq K_{0} / 2(2 s+1)^{v}$ and let

$$
\tau=K_{0} \beta / 2(2 s+1)^{\nu} .
$$

Then the Peierls condition holds for sufficiently large $\beta$. In order to show the regularity condition in Assumption 1.2, we choose

$$
\delta(x, y)=\varepsilon \log (1+\|x-y\|), \quad J(\|x-y\|)=\beta c\|x-y\|^{-\lambda} .
$$

Then the conditions in (1.3) and (1.4) hold. By Assumption 5.1 we also obtain

$$
\begin{aligned}
2 \sum_{r=0}^{\infty} \sum_{y \in \mathbb{Z}^{v}} J(2 s+r+\|y\|) & \leqq 2 c \beta \sum_{r=0}^{\infty} \sum_{y \in \mathbb{Z}^{v}}(2 s+r+\|y\|)^{-\lambda} \\
& \leqq \text { const. } \beta s^{-(v+1 / 2)}
\end{aligned}
$$

Thus we first choose $s$ so large that (1.5) in Assumption 1.2 holds and then choose $\beta$ sufficiently large. Then the Peierls condition holds. Thus Theorem 5.2 follows from Theorem 1.3.

We next consider more general interactions. We write that

$$
K_{1} \equiv c \sum_{r=0}^{\infty} \sum_{x \in \mathbb{Z}^{v}}(2+r+\|x\|)^{-\lambda}+\sum_{\substack{\|x\|=1 . \\|x| \neq 1}}|K(\|x\|)|,
$$

where $c$ is the constant in Assumption 5.1.

Theorem 5.3. Let $K(x-y)$ satisfy the bound in Assumption 5.1. Assume that $K_{0}>0$ and $K_{1} \leqq K_{0} / 196(3)^{v}$. Then the results in Theorem 1.3 hold for sufficiently large $\beta$. Proof. Choose $s=1$. Then by (5.7) it is easy to show that

$$
\Phi_{0}(\Gamma) \geqq\left(3^{-v} K_{0}-K_{1}\right) \beta|\Gamma|,
$$

so by (5.11) we have

$$
\Phi(\Gamma) \geqq\left(3^{-v} K_{0}-K_{1}-|\lambda|\right) \beta|\Gamma|
$$


Thus the Peierls condition is satisfied for sufficiently small $|\lambda|\left(\leqq K_{0} / 2\left(3^{v}\right)\right)$. Notice that

$$
2 \sum_{r=0}^{\infty} \sum_{x \in \mathbb{Z}^{v}} J(2+r+\|x\|) \leqq 2 \beta K_{1} .
$$

By choosing $\tau=\left(K_{0} 3^{-v} / 3\right) \beta$, we see that the regularity condition also holds.

Remark. The conditions in Theorem 5.2 and Theorem 5.3 are obviously not optimal. We only tried to give general ideas how one can apply our results to specific models.

\section{Appendix: Proof of Proposition 4.2}

We produce the proof of Proposition 4.2 in this appendix. We first describe general ideas of the proof. For any $q$-contour $\Gamma=\left(M, \omega_{M}\right)$ let $\Phi_{1}(\Gamma ; \omega)$ be the one-body contour interaction defined in (2.2.6) of Part I:

$$
\begin{aligned}
\Phi_{1}(\Gamma ; \omega)= & \sum_{A \subset V(\Gamma)}\left[\Phi_{A}\left(\omega_{A}\right)-\Phi_{A}\left(\omega_{A, q}\right)\right] \\
& +\sum_{\{x, y\} \cap V(\Gamma) \neq \phi}\left[J_{x-y}\left(\omega_{q}(x ; V(\Gamma)), \omega_{q}(y ; V(\Gamma))-J_{x-y}(q, q)\right],\right.
\end{aligned}
$$

where for any $B \subset \mathbb{Z}^{v}$ and $\omega \in \Omega^{B}$,

$$
\omega_{q}(x, B)=\left\{\begin{array}{lll}
\omega(x) & \text { if } & x \in B \\
q & \text { if } & x \notin B
\end{array} .\right.
$$

We will decompose $\Phi_{1}(\Gamma ; \omega)$ by

$$
\Phi_{1}(\Gamma ; \omega)=\Phi(\Gamma)+\sum_{q^{\prime} \in Q}\left(e_{q^{\prime}}-e_{q}\right)\left|\operatorname{Int}_{q^{\prime}} \Gamma\right|+\sum_{q^{\prime} \in Q} H\left(\operatorname{Int}_{q^{\prime}} \Gamma ; \omega\right)+\text { Remainder, }
$$

where for any configuration $\omega$ on $\operatorname{Int}_{q^{\prime}} \Gamma$ with $\partial \equiv \operatorname{ext}(\partial \omega) \in \mathscr{E}_{q^{\prime}}\left(\operatorname{Int}_{q^{\prime}} \Gamma\right)$, $H\left(\operatorname{Int}_{q^{\prime}} \Gamma ; \omega\right)=H(\partial ; \omega)$. We will show that

$$
\mid \text { Remainder }|\leqq \tau| \Gamma \mid / 6 .
$$

Then from (2.4), (A.3) and (A.4) we obtain

$$
\begin{aligned}
Z_{q}(\Gamma) \leqq & \left\{\prod_{q^{\prime} \in Q} Z_{q^{\prime}}\left(\operatorname{Int}_{q^{\prime}} \Gamma\right)\right\} \exp (\tau|\Gamma| / 6) \\
& \cdot \exp \left\{-\Phi(\Gamma)-\sum_{q^{\prime} \in Q}\left(e_{q^{\prime}}-e_{q}\right)\left|\operatorname{Int}_{q^{\prime}} \Gamma\right|\right\} .
\end{aligned}
$$

Next, for any $\partial \in \mathscr{E}_{q}(\operatorname{Int} \Gamma)$, let $\partial=\bigcup_{q^{\prime} \in Q} \partial_{q^{\prime}}, \partial_{q^{\prime}} \in \mathscr{E}_{q}\left(\operatorname{Int}_{q^{\prime}} \Gamma\right)$. Writing

$$
H(\partial ; \omega)=\sum_{q^{\prime} \in Q} H\left(\partial_{q^{\prime}} ; \omega\right)+\sum_{\substack{q^{\prime}, q^{\prime \prime} \in Q \\ q^{\prime} \neq q^{\prime \prime}}} \Phi_{2}\left(\partial_{q^{\prime}}, \partial_{q^{\prime \prime}} ; \omega\right),
$$

and using Proposition 4.2.2(a) of Part I to obtain

$$
\sum_{q^{\prime}, q^{\prime \prime} \in Q}\left|\Phi_{2}\left(\partial_{q^{\prime}}, \partial_{q^{\prime \prime}} ; \omega\right)\right| \leqq \sum_{q^{\prime} \in Q} \tau\left|\mathrm{bd}\left(\operatorname{Int}_{q^{\prime}} \Gamma\right) / 48 \leqq \tau\right| \Gamma \mid / 48,
$$


we conclude that

$$
Z_{q}(\operatorname{Int} \Gamma) \geqq\left\{\prod_{q^{\prime} \in Q} Z_{q}\left(\operatorname{Int}_{q^{\prime}} \Gamma\right)\right\} \exp (-\tau|\Gamma| / 48)
$$

Thus the proposition will follow from (A.5), (A.8) and the Peierls condition (Assumption 1.1).

In the rest of the appendix we establish (A.3) and (A.4), and so complete the proof of the proposition. Using the expression (A.1) and the fact that $\Phi_{A}\left(\omega_{A}\right)-\Phi_{A}\left(\omega_{A, q}\right)=0$ if $A \cap V(\Gamma)^{c} \neq \phi$, we can write that for any $q$-contour $\Gamma=\left(M, \omega_{M}\right)$,

$$
\Phi_{1}(\Gamma ; \omega)=\Phi(\Gamma)+\Phi_{2}(\operatorname{Int} \Gamma ; \omega)+\Phi_{3}(\Gamma ; \omega)
$$

where $\Phi(\Gamma)$ be the contour functional defined in (1.2), and

$$
\begin{aligned}
\Phi_{2}(\operatorname{Int} \Gamma ; \omega)= & \sum_{A \cap \operatorname{Int} \Gamma \neq \phi} \frac{|A \cap \operatorname{Int} \Gamma|}{|A|}\left[\Phi_{A}\left(\omega_{A}\right)-\Phi_{A}\left(\omega_{A, q}\right)\right] \\
& +\sum_{\substack{\{x, y\} \cap \operatorname{Int} \Gamma \neq \neq \\
\|x-y\| \leqq s}} \frac{|\{x, y\} \cap \operatorname{Int} \Gamma|}{2}\left[J_{x-y}(\omega(x), \omega(y))-J_{x-y}(q, q)\right], \\
\Phi_{3}(\Gamma ; \omega)= & \sum_{\substack{\{x, y\} \cap V(\Gamma) \neq \phi \\
|x-y|>s}}\left[J_{x-y}\left(\omega_{q}(x ; V(\Gamma)), \omega_{q}(y ; V(\Gamma))\right)-J_{x-y}(q, q)\right] .
\end{aligned}
$$

For typological simplification of notation, we use the following abbreviated notations: For any $q$-contour $\Gamma=\left(M, \omega_{M}\right)$ denote

$$
\begin{aligned}
& I_{q^{\prime}} \equiv \operatorname{Int}_{q^{\prime}} \Gamma, \quad \text { Int } \Gamma=\bigcup_{q^{\prime} \in Q} I_{q^{\prime}}, \\
& \sum_{\{x, y\} \cap I_{q^{\prime}} \neq \phi}^{\prime}=\sum_{\substack{\{x, y\} \cap I_{q^{\prime}} \neq \phi \\
|x-y| \leqq s}}, \quad \sum_{\{x-y\} \cap I_{q} \neq \phi}^{\prime \prime}=\sum_{\substack{\{x, y\} \cap I_{q} \neq \phi \\
\|x-y\|>s}} .
\end{aligned}
$$

Then from (A.9) it follows that

where

$$
\Phi_{2}(\operatorname{Int} \Gamma ; \omega)=\tilde{\Phi}_{2}(\operatorname{Int} \Gamma ; \omega)+E_{2}(\operatorname{Int} \Gamma),
$$

$$
\begin{aligned}
\tilde{\Phi}_{2}(\operatorname{Int} \Gamma ; \omega)= & \sum_{q^{\prime} \in Q} \sum_{A \cap I_{q^{\prime}} \neq \phi} \frac{\left|A \cap I_{q^{\prime}}\right|}{|A|}\left[\Phi_{A}\left(\omega_{A}\right)-\Phi_{A}\left(\omega_{A, q^{\prime}}\right)\right] \\
& +\sum_{q^{\prime} \in Q} \sum_{\{x, y\} \cap I_{q^{\prime}} \neq \phi} \frac{\left|\{x, y\} \cap I_{q^{\prime}}\right|}{2}\left[J_{x-y}(\omega(x), \omega(y))-J_{x-y}\left(q^{\prime}, q^{\prime}\right)\right],
\end{aligned}
$$

$$
\begin{aligned}
E_{2}(\operatorname{Int} \Gamma)= & \sum_{q^{\prime} \in Q} \sum_{A \cap I_{q^{\prime}} \neq \phi} \frac{\left|A \cap I_{q^{\prime}}\right|}{|A|}\left[\Phi_{A}\left(\omega_{A, q^{\prime}}\right)-\Phi\left(\omega_{A, q}\right)\right] \\
& +\sum_{q^{\prime} \in Q} \sum_{\{x, y\} \cap I_{q^{\prime}} \neq \phi}^{\prime} \frac{\left|\{x, y\} \cap I_{q^{\prime}}\right|}{2}\left[J_{x-y}\left(q^{\prime}, q^{\prime}\right)-J_{x-y}(q, q)\right] .
\end{aligned}
$$


We next consider $\Phi_{3}(\Gamma ; \omega)$ given in (A.11). We decompose $\Phi_{3}(\Gamma ; \omega)$ by

$$
\Phi_{3}(\Gamma ; \omega)=\Phi_{3}^{1}(\operatorname{Int} \Gamma ; \omega)+\Phi_{3}^{2}(\Gamma ; \omega),
$$

where

$$
\begin{aligned}
\Phi_{3}^{1}(\operatorname{Int} \Gamma ; \omega)= & \sum_{\substack{\{x, y\} \cap \operatorname{Int} \Gamma \neq \phi \\
x-y \mid>s}}\left[J_{x-y}\left(\omega_{q}(x ; V(\Gamma)), \omega_{q}(y ; V(\Gamma))\right)-J_{x-y}(q, q)\right] \\
= & \sum_{q^{\prime} \in Q} \sum_{\{x, y\} \cap I_{q^{\prime}} \neq \phi}^{\prime \prime}\left[J_{x-y}\left(\omega_{q}(x ; V(\Gamma)), \omega_{q}(x ; V(\Gamma))\right)-J_{x-y}(q-q)\right] \\
& -\sum_{\substack{q^{\prime}, q^{\prime \prime} \in Q \\
q^{\prime} \neq q^{\prime \prime}}} \sum_{\substack{x \in I^{\prime} \\
y \in q_{q^{\prime \prime}}}} \frac{1}{2}\left[J_{x-y}(\omega(x), \omega(y))-J(q, q)\right], \\
\Phi_{3}^{2}(\Gamma ; \omega)= & \sum_{\substack{\{x, y\} \cap M \neq \phi: \\
\{x, y\} \cap \ln t \Gamma=\phi \\
|x-y|>s}}\left[J_{x-y}\left(\omega_{q}(x ; V(\Gamma)), \omega_{q}(y ; V(\Gamma))\right)-J_{x-y}(q, q)\right] .
\end{aligned}
$$

Combining (A.9), (A.13) and (A.16) we arrive at

$$
\Phi_{1}(\Gamma ; \omega)=\Phi(\Gamma)+\tilde{\Phi}_{2}(\operatorname{Int} \Gamma ; \omega)+E_{2}(\operatorname{Int} \Gamma)+\Phi_{3}^{1}(\operatorname{Int} \Gamma ; \omega)+\Phi_{3}^{2}(\Gamma ; \omega) .
$$

Finally we decompose $\Phi_{3}^{1}($ Int $\Gamma ; \omega)$ in the following way: By (A.17) it can be written

$$
\Phi_{3}^{1}(\operatorname{Int} \Gamma ; \omega)=\sum_{i=1}^{4} \Phi_{3, i}^{1}(\operatorname{Int} \Gamma ; \omega)
$$

where

$$
\begin{aligned}
\Phi_{3,1}^{1}(\operatorname{Int} \Gamma ; \omega)= & \sum_{q^{\prime} \in Q} \sum_{\{x, y\} \cap I_{q^{\prime}} \neq \phi}^{\prime \prime}\left[J_{x-y}\left(\omega_{q^{\prime}}\left(x ; I_{q^{\prime}}\right), \omega_{q^{\prime}}\left(y ; I_{q^{\prime}}\right)\right)-J_{x-y}\left(q^{\prime}, q^{\prime}\right)\right], \\
\Phi_{3,2}^{1}(\operatorname{Int} \Gamma ; \omega)= & \sum_{q^{\prime} \in Q} \sum_{\{x, y\} \cap I_{q^{\prime}} \neq \phi}^{\prime \prime}\left\{J _ { x - y } \left(\omega_{q}(x ; V(\Gamma)), \omega_{q}(y ; V(\Gamma))\right.\right. \\
& \left.-J_{x-y}\left(\omega_{q^{\prime}}\left(x ; I_{q^{\prime}}\right), \omega_{q^{\prime}}\left(y ; I_{q^{\prime}}\right)\right)\right\} \\
\Phi_{3,3}^{1}(\operatorname{Int} \Gamma ; \omega)= & \sum_{q^{\prime} \in Q} \sum_{\{x, y\} \cap I_{q^{\prime}} \neq \phi}^{\prime \prime}\left[J_{x-y}\left(q^{\prime}, q^{\prime}\right)-J_{x-y}(q, q)\right] \\
\Phi_{3,4}^{1}(\operatorname{Int} \Gamma ; \omega)= & -\frac{1}{2} \sum_{\substack{q^{\prime}, q^{\prime \prime} \in Q \\
q^{\prime} \neq q^{\prime \prime}}} \sum_{\substack{x \in I_{q^{\prime}} \\
y \in I_{q^{\prime \prime}}}}\left[J_{x-y}\left(q^{\prime}, q^{\prime}\right)-J_{x-y}(q, q)\right]
\end{aligned}
$$

Recall $I_{q^{\prime}}=\operatorname{Int}_{q^{\prime}} \Gamma$. For any $\omega \in \Omega^{I_{q^{\prime}}}$ with $\partial=\operatorname{ext}(\partial \omega) \in \mathscr{E}_{q^{\prime}}\left(I_{q^{\prime}}\right)$ denote

$$
H\left(\operatorname{Int}_{q^{\prime}} \Gamma ; \omega\right)=H\left(\omega_{I_{q^{\prime}}}, \omega_{I_{q^{\prime}, q^{\prime}}^{c}}\right)-H\left(\omega_{I_{q^{\prime}, q^{\prime}}} \mid \omega_{I_{q^{\prime}}^{c}, q^{\prime}}\right)=H(\partial ; \omega) \text {. }
$$

Notice that for any $\omega \in \Omega(\Gamma), \Phi_{A}\left(\omega_{A}\right)-\Phi_{A}\left(\omega_{A, q^{\prime}}\right)=0$ if $\operatorname{diam}(A) \leqq s$ and $A \cap I_{q^{\prime}}^{c} \neq \phi$. Thus from (A.14), (A.19) and (A.25) it follows that

$$
\tilde{\Phi}_{2}(\operatorname{Int} \Gamma ; \omega)+\Phi_{3,1}^{1}(\operatorname{Int} \Gamma ; \omega)=\sum_{q^{\prime} \in Q} H\left(\operatorname{Int}_{q^{\prime}} \Gamma ; \omega\right) \text {. }
$$

Consider $\Phi_{3,3}^{1}(\operatorname{Int} \Gamma ; \omega)$ in $(\mathrm{A} .23)$. We write

$$
\Phi_{3,3}^{1}(\operatorname{Int} \Gamma ; \omega)=E_{3}^{1}(\operatorname{Int} \Gamma)+E_{3}^{2}(\operatorname{Int} \Gamma),
$$


where

$$
\begin{aligned}
& E_{3}^{1}(\operatorname{Int} \Gamma)=\sum_{q^{\prime} \in Q} \sum_{\{x, y\} \cap I_{q^{\prime}} \neq \phi}^{\prime \prime} \frac{|\{x, y\} \cap M|}{2}\left\{J_{x-y}\left(q^{\prime}, q^{\prime}\right)-J_{x-y}(q, q)\right\} \\
& E_{3}^{2}(\operatorname{Int} \Gamma)=\sum_{q^{\prime} \in Q} \sum_{\substack{x \in I_{q^{\prime}} \\
y \in I_{q^{\prime}}^{c^{\prime}} \\
\| x, y-y \mid>s}} \frac{1}{2}\left\{J_{x-y}\left(q^{\prime}, q^{\prime}\right)-J_{x-y}(q, q)\right\}
\end{aligned}
$$

Using the definition of the specific energy $e_{q}$ in (1.6) one can check that for any finite $\Lambda \subset \mathbb{Z}^{v}$

$$
e_{q}|\Lambda|=\sum_{A \cap \Lambda \neq \phi} \frac{|A \cap \Lambda|}{|A|} \Phi_{A}\left(\omega_{A, q}\right)+\sum_{\{x . y\} \cap \Lambda \neq \phi} \frac{|\{x, y\} \cap \Lambda|}{2} J_{x-y}(q, q)
$$

Thus from (A.15), (A.28) and (A.30) it follows that

$$
E_{2}(\operatorname{Int} \Gamma)+E_{3}^{1}(\operatorname{Int} \Gamma)=\sum_{q^{\prime} \in Q}\left(e_{q^{\prime}}-e_{q}\right)\left|\operatorname{Int}_{q^{\prime}} \Gamma\right| .
$$

Using the regularity condition (Assumption 1.2) one obtains

$$
\begin{aligned}
\left|E_{3}^{2}(\operatorname{Int} \Gamma)\right| & \leqq \sum_{q^{\prime} \in Q} \sum_{\substack{x \in I_{q^{\prime}} \\
y \in I_{q^{\prime}}}} J(2 s+\|x-y\|) \\
& \leqq \sum_{q^{\prime} \in Q} \tau\left|\operatorname{bd}\left(I_{q^{\prime}}\right)\right| / 48 \leqq \tau|\Gamma| / 48
\end{aligned}
$$

Again, we use the regularity condition, the method similar to that used above, and the fact that

$\left.J_{x-y}\left(\omega_{q}(x ; V(\Gamma)), \omega_{q}(y ; V(\Gamma))\right)-J_{x-y}\left(\omega_{q^{\prime}}\left(x ; I_{q^{\prime}}\right), \omega_{q^{\prime}}\left(y ; I_{q^{\prime}}\right)\right)\right)=0 \quad$ if $\quad\{x, y\} \subset I_{q^{\prime}}$ to obtain the following bounds:

$$
\begin{aligned}
\left|\Phi_{3}^{2}(\Gamma ; \omega)\right| & \leqq \tau|\Gamma| / 48, \\
\left|\Phi_{3,2}^{1}(\operatorname{Int} \Gamma ; \omega)\right| & \leqq \sum_{q^{\prime} \in Q} \tau\left|\mathrm{bd}\left(I_{q^{\prime}}\right)\right| / 48 \leqq \tau|\Gamma| / 48, \\
\left|\Phi_{3,4}^{1}(\operatorname{Int} \Gamma ; \omega)\right| & \leqq \tau|\Gamma| / 48 .
\end{aligned}
$$

We now combine (A.19), (A.20), (A.26), (A.27), (A.31), (A.32) and (1.32) to conclude that

$$
\begin{aligned}
\Phi_{1}(\Gamma ; \omega) \geqq & \Phi(\Gamma)+\sum_{q^{\prime} \in Q} H\left(\operatorname{Int}_{q^{\prime}} \Gamma ; \omega\right) \\
& +\sum_{q^{\prime} \in Q}\left(e_{q^{\prime}}-e_{q}\right)\left|\operatorname{Int}_{q^{\prime}} \Gamma\right|-\tau|\Gamma| / 12 .
\end{aligned}
$$

This proved (A.3) and (A.4) and so we complete the proof of the proposition.

Acknowledgements. I should like to express my gratitude to the referee, for his suggestions, and to thank the Korean Ministry of Education for financial support. 


\section{References}

1. Bricmont, J., Kuroda, K., Lebowitz, J. L.: First order phase transitions in lattice and continuous systems: Extension of Pirogov-Sinai theory, Commun. Math. Phys. 101, 501-538 (1985)

2. Dobrushin, R. L., Zahradnik, M.: Phase diagrams for continuous spin models: Extension of PirogovSinai theory, Preprint

3. Park, Y. M.: Extension of the Pirogov-Sinai theory of phase transitions to infinite range interactions. I. Cluster expansion. Commun. Math. Phys. 114, 187-218 (1988)

4. Pirogov, S. A., Sinai, Ya. G.: Phase diagram of classical spin systems I and II. Theor. Math. Phys. 25, 1185 (1976) and 26, 39 (1976)

5. Sinai, Ya. G.: Theory of phase transition: Rigorous results. New York: Pergamon Press 1982

6. Zahradnik, M.: An alternative version of Pirogov-Sinai theory. Commun. Math. Phys. 93, 559 (1984)

Communicated by J. Fröhlich

Received February 26, 1987; in revised form May 28, 1987 
